\title{
OTIOTOMICS
}

Revista de economía, empresa y sociedad

\section{PMIMSGAT 2.0: e-learning y TIC como factores de competitividad}

Joan Manzanares Morales

Técnico de gestión académica

Estudios de Economía y Empresa (UOC)

RESUMEN Proyecto final del máster de Educación y TIC de la UOC, que se ha elaborado siguiendo el itinerario formativo de especialización de dirección y gestión de proyectos e instituciones con aplicación de las TIC.

El proyecto PYMESCAT 2.0 tiene como objetivos generales crear una plataforma de encuentro del tejido empresarial de PYMES catalanas para realizar acciones de formación conjunta, así como llevar a cabo el curso de formación en TIC para trabajadores de PYMES para la mejora de su competitividad.

Este proyecto se ha desarrollado siguiendo el modelo ADDIE que queda reflejado en la estructura de la memoria del proyecto, donde hay explicadas cada una de las fases: análisis, diseño, desarroIlo, implementación y evaluación del proyecto. En el curso diseñado se ha desarrollado e implementado una unidad de muestra dentro de un entorno Moodle, concretamente la unidad 7, que hace referencia al diseño y uso de blogs. El modelo pedagógico en el cual se basa este proyecto es el learning by doing ('aprendizaje por medio de la práctica').

PALABRAS CLAVE e-learning; TIC; PYMES; competitividad; Moodle; learning by doing; modelo ADDIE

\section{PIMESCAT 2.0: E-learning and ICT as Competitiveness Factors}

ABSTRACT This article explains my final project of the Master in Education and ICT of the UOC (Catalan Open University), which has been prepared following the training program in management projects and organizations with the application of ICT.

The project PIMESCAT 2.0 aims to create a meeting platform for the Catalan SMEs to carry out joint training and conduct ICT training courses for employees of SMEs, which contribute to improve their competitiveness.

This project has been developed following the ADDIE model, as reflected in the structure of the project report, where each phase is explained: Analysis, Design, Development, Implementation and Evaluation of the project. In this course I have developed and implemented a sample unit within 
a Moodle environment, more specifically, unit 7, dedicated to the design and use of blogs. The pedagogical model of this project is based in learning by doing.

KEYWORDS e-learning; ICT; SMEs; competitiveness; Moodle; learning by doing; ADDIE model

\section{Introducción}

Este artículo es un resumen de la memoria ${ }^{1}$, que recoge mi proyecto final de máster universitario de Educación y TIC de la UOC. El trabajo se elaboró siguiendo el itinerario formativo de especialización en dirección y gestión de proyectos e instituciones con aplicación de las TIC.

La idea del proyecto surge de la identificación, a partir de mi conocimiento por temas profesionales, de las necesidades que hoy en día tienen tanto los profesionales que trabajan en diferentes empresas, de las necesidades formativas que tienen las PYMES y de las dificultades que tienen para llevarlas a cabo, como también de mi conocimiento sobre las TIC y de sus ventajas en la sociedad de la información. Es por este motivo que quise contextualizar el proyecto dentro de las PYMES en mi entorno más cercano y hacer un curso para adquirir habilidades y competencias en TIC.

PYMESCAT 2.0 es el título del proyecto que se presenta a continuación y tiene como objetivos generales crear una plataforma de encuentro del tejido empresarial de PYMES catalanas para realizar acciones de formación conjunta y llevar a cabo el curso de formación en TIC para trabajadores de PYMES para la mejora de su competitividad.

\section{Análisis de necesidades y definición de los objetivos}

Este proyecto de final de máster no se desarrolla dentro de un contexto acotado, como puede ser dentro de una organización, sino que pretende dar respuesta a las necesidades de un contexto más amplio, como son las PYMES. Por este motivo, la recogida y análisis de necesidades, la definición de público objetivo y la definición de los objetivos y resultados esperados son clave para el desarrollo y viabilidad de este proyecto.

Para la obtención de los datos, elaboré un formulario en línea donde se recogían una serie de ítems, para obtener información sobre el uso de las TIC, las necesidades formativas vinculadas a las TIC, y el perfil del público objetivo y del entorno profesional donde trabajan. Pedí la colaboración de amigos y familiares que trabajasen en PYMES, para que, además de responder el formulario, lo hicieran llegar a sus amigos, compañeros de trabajo y familiares que trabajasen en PYMES.

De les encuestas recibidas extraje distinta información y datos que sirvieron como base para la definición de los objetivos, los más relevantes de los cuales son:

- El $70 \%$ eran trabajadores del sector del comercio y la hostelería.

- El 80\% tenía entre el 25 y 35 años.

- El $72 \%$ dijo que el uso de las TIC en el trabajo es bajo, pero de estos, el $60 \%$ en su vida personal hace un uso mediano de las TIC.

- El 68\% ha solicitado formación en blogs / páginas web para su desarrollo profesional, y el $75 \%$ para su vida personal.

- De las personas que solicitaron formación en páginas webs / blogs, el 90\% tiene como principal objetivo la promoción de la empresa en la red y llegar a sus clientes potenciales de una forma más económica y directa.

- Un 52\% afirma que su empresa tiene problemas de competitividad en el mercado.

1. Memoria depositada en el Repositorio Institucional de la UOC (O2) <http://hdl.handle.net/10609/26741>, el portal que recoge, difunde y preserva las publicaciones digitales en acceso abierto de los miembros de la UOC, elaborados en el desarrollo de sus actividades de investigación, de docencia y de gestión. 
Con la recogida y análisis de los datos obtenidos, pude definir el público objetivo (trabajadores/as de 25 a 35 años, del sector industrial y servicios del ámbito catalán), las necesidades formativas de este público objetivo y también la definición de los objetivos del proyecto.

\section{Objetivos generales del proyecto}

- Crear una plataforma de encuentro del tejido empresarial de PYMES catalanas, para llevar a cabo acciones de formación conjunta.

- Llevar a cabo el curso PYMESCAT 2.0 de formación en TIC para trabajadores de PYMES.

\section{Objetivos específicos del proyecto}

- Asesoramiento profesional para el desarrollo de acciones formativas virtuales.

- Mejorar la competitividad de las PYMES.

- Detectar las necesidades formativas del mercado.

- Establecer marketing relacional con las empresas-cliente.

- Difundir los cursos elaborados.

- Reciclar y actualizar los conocimientos ofimáticos y de internet de los trabajadores de PYMES en Cataluña.

- Formar a los profesionales en/con las TIC, y en concreto en las aplicaciones 2.0, para la adquisición de competencias que mejoren su desarrollo profesional.

- Fomentar el trabajo en red.

- Fomentar el trabajo colaborativo, transversal y multidisciplinar.

- Conectar los diferentes profesionales con sus compañeros de trabajo o proveedores y clientes externos.

- Establecer mecanismos de comunicación transversal y horizontal dentro de las PYMES.

- Crear un sistema de reciprocidad y de retroalimentación para la mejora de los procesos internos y externos, utilizando las TIC.

En la definición de los objetivos, fui muy cuidadoso en definirlos tan reales y factibles como fuera posible, y que por lo tanto, que fueran alcanzables, como también coherentes y complementarios entre sí, y que pudieran ser evaluados.

Con los objetivos definidos, y para definir una estrategia, elaboré una matriz DAFO donde se recogían todos los factores internos y externos que influían en el proyecto, y que por lo tanto, nos daba una visión global de la situación del proyecto dentro del contexto definido, para tomar medidas y estrategias necesarias para garantizar su éxito.

\section{Diseño y desarrollo}

Siguiendo el modelo ADDIE ${ }^{2}$ de diseño instruccional, después de definir a quién iba dirigido y cuáles eran los objetivos, se debía iniciar el diseño y desarrollo del curso.

En primer lugar, decidí en qué modelo pedagógico estaría basado, en este caso en el modelo learning by doing, es decir, en un aprendizaje basado en la práctica durante el curso. Esto quiere decir que el material y los recursos presentados son muy importantes, pero lo más importante es el diseño de las actividades, para que los estudiantes puedan aplicar los conceptos y recursos presentados para adquirir las competencias marcadas.

2. El modelo ADDIE es un proceso de diseño instruccional interactivo, donde los resultados de la evaluación formativa de cada fase pueden conducir al diseñador instruccional de retorno a cualquiera de las fases previas. El producto final de una fase es el producto de inicio de la siguiente fase. Véase: Steven J. McGriff (2000). Instructional System Design (ISD): Using the ADDIE Model. College of Education, Penn State University [en línea] [Consulta: 20 de enero de 2014]. Disponible en: <http://disenoinstrucional.files.wordpress.com/2007/09/addiemodel.doc>. 
Este fue momento clave del proyecto, porque si no tenía bien definido el público objetivo y los objetivos marcados, podría haber desviaciones en el momento de hacer el diseño, y caer en la trampa de condicionar el diseño a la plataforma tecnológica (Moodle) que utilizaría. Por lo tanto, todo tenía que estar relacionado y ser coherente. Es decir, los objetivos con las competencias, las actividades con las competencias, el material con las actividades y el método de evaluación con los objetivos, actividades y competencias.

El curso se divide en 8 módulos, que se llevarían a cabo durante 3 meses (150h). De todas formas, siempre hay la posibilidad de añadir o quitar módulos según las necesidades de los estudiantes. Por otro lado, de estos 8 módulos solo desarrollé uno como muestra, el modulo 7 , ya que al ser un proyecto de dirección, no me centré tanto en el desarrollo de todo el curso, sino en cómo llevar a cabo la implantación de este modulo de muestra.

Se definieron elementos básicos para el diseño de este entorno de aprendizaje: organización de la información (estructura), aspectos motivacionales, interactividad, multimedia, hipertexto, navegabilidad, interfaz, usabilidad, accesibilidad, y flexibilidad. Todos estos aspectos tenían que dar respuesta a las necesidades de los diferentes usuarios de la plataforma: estudiantes, visitantes, docentes y administradores.

\section{Implementación}

Sobre el papel, ya lo tenía todo diseñado, planificado y presupuestado; ahora era necesario implementarlo y ver la viabilidad real del proyecto. Como ya he comentado anteriormente, para el proyecto solo implementaría una unidad de muestra, y a partir de esta unidad y del test de usuario que realizaría posteriormente, podría mejorar esta unidad y por extensión el resto del curso antes de ser desarrollado e implementado al 100\%, para intentar minimizar riesgos.

Aunque fue una implementación parcial, que no se llegó a comercializar, mediante el test de usuario pude poner el proyecto en una simulación real, donde comprobé que es muy difícil planificarlo todo, y vi que hasta que el proyecto no se lleva a la práctica no puedes descubrir ciertas variables que influyen y que no podemos controlar, pero que hay que tener presentes para poderles dar respuesta.

Además de implementar la unidad de muestra, realicé un blog para la difusión del proyecto, para una posible comercialización del mismo. Además, en el mismo Moodle del curso, creé un apartado para fomentar el contacto entre PYMES y la demanda y petición de nuevos cursos basados en las TIC, compartiendo a la vez los costes de los mismos.

\section{Evaluación}

En todo proyecto formativo, la evaluación es un elemento que se encuentra en todo el proceso (diagnóstica, formativa y sumativa), pero especialmente con proyectos de simulación, ya que aparte de evaluar y mejorar, es una fuente de información muy importante. Todas las evaluaciones hechas se han realizado a través de un formulario en línea y mediante Googledocs, así como los cuestionarios a los estudiantes, al iniciar el curso y al finalizar cada módulo y también los cuestionarios de calidad dirigidos a las empresas clientes.

En cambio, en el test de usuario, la evaluación se hizo de forma presencial para poder evaluar aspectos que un cuestionario en línea no podía recoger.

En general se obtuvo una nota aceptable para ser una prueba piloto, pero se debían mejorar ciertos aspectos que, con más tiempo, y con un presupuesto real, serian factibles. Sobre todo el proyecto se vio limitado por la plataforma gratuita Moodle, con la cual no se pudo desarrollar tal y como estaba planificado, sobre todo la parte de usabilidad y de interacción, la cual era muy importante con participantes que muy probablemente tenían poca experiencia en formación virtual.

Las encuestas de calidad del curso iban dirigidas tanto a los estudiantes (una al finalizar el curso y otra al cabo de 3 meses, para verificar la aplicabilidad y transferencia de las competencias adquiridas en su día a día profesional), como también una encuesta a las organizaciones en las cuales trabajan los estudiantes. 


\section{Conclusiones}

En un proyecto que no se lleva a la realidad, es necesario tener muy presente tres elementos clave: un buen análisis de las necesidades, una coherencia interna entre las diferentes partes del proyecto y realizar simulaciones o test de usuarios que nos permitan jugar con la simulación y la realidad. De esta forma, nos permitirá tener un proyecto concebido en un contexto de laboratorio, pero con la máxima información para acercarlo a la realidad. La duda es saber si este proyecto habría sido viable cuando se hubiera desarrollado e implementado al 100\% para su comercialización, y si los resultados obtenidos serían igual de buenos que con el test de usuario que realicé.

En relación con el desarrollo del proyecto, su realización fue todo un reto y un gran esfuerzo, sobre todo por tratarse de una simulación en la creación de una empresa y el desarrollo de un curso en línea. Como ya he comentado anteriormente, una de les fases a la cual dediqué más tiempo fue en el análisis de las necesidades y la definición de objetivos, para minimizar la distancia entre mi proyecto simulado y el contexto real. Durante el semestre, dentro de la evaluación formativa y siguiendo el calendario planificado, tenía que ir entregando las diferentes fases cumplimentadas para que el consultor las evaluara. De esta forma, durante el desarrollo del proyecto ya podía ir corrigiendo y mejorando diferentes aspectos, pero esto implicaba que tenía que modificar sobre la marcha la fase en curso para recoger los cambios de la anterior fase, pero teniendo presente la siguiente fecha de entrega. El feedback con el consultor, y también con los demás compañeros, fue muy intenso y enriquecedor, lo cual me permitió una evaluación exhaustiva entre los compañeros de aula, así como también evaluar al consultor y la asignatura de trabajo final.

Para llevar a cabo este tipo de proyectos se recomienda utilizar herramientas gratuitas para el desarrollo del blog de la empresa y del curso en línea. Ahora bien, recientemente he podido comprobar que la plataforma Moodle freewebclass ya no existe, y que por lo tanto, todo lo que tenía implementado en el marco del proyecto ya no es accesible. Sin embargo, en la memoria del proyecto hay capturas de pantalla y explicaciones de cada uno de los diferentes espacios del entorno Moodle que alojaba la unidad de muestra. Este hecho me hará reflexionar, de cara a futuros proyectos, sobre las ventajas e inconvenientes de utilizar herramientas TIC gratuitas.

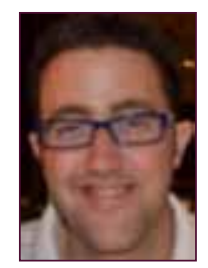

Joan Manzanares Morales

jmanzanaresmo@uoc.edu

Técnico de gestión académica

\section{Estudios de Economía y Empresa (UOC)}

Licenciado en Pedagogía por la Universitat de Barcelona, graduado superior en Formación en las Organizaciones por la Universitat de Barcelona, máster en Dirección de marketing y comunicación por la UOC, máster universitario en Educación y TIC (e-learning) por la UOC. Desde 2006, forma parte del personal de gestión de la UOC y actualmente, desde 2009, desarrolla su tarea profesional como técnico de gestión académica en los Estudios de Economía y Empresa de la UOC.

Los textos publicados en esta revista están sujetos -si no se indica lo contrario- a una licencia de Reconocimiento 3.0 España de Creative Commons. Puede copiarlos, distribuirlos, comunicarlos públicamente, hacer obras derivadas y usos comerciales siempre que reconozca los créditos de las obras (autoría, nombre de la revista, institución editora) de la manera especificada por los autores o por la revista. La licencia completa se puede consultar en http://creativecommons.org/licenses/ by/3.0/es/deed.es.

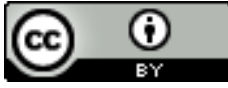

